Recepción: 20 / 04 / 2017

Aceptación: 20 / 05 / 2017

Publicación: 15 / 09 / 2017
Ciencias Químicas

Artículo Científico

\title{
Estudio comparativo del rendimiento del furfural a partir de diferentes residuos agrícolas (Cascarilla de Arroz, Bagazo de Caña, Zuro de Maíz)
}

Comparative study of the performance of furfural from different agricultural residues (Cascarilla de Arroz, Bagasse of Caña, Zuro de Maíz)

\section{Estudo comparativo do desempenho furfural de diferentes resíduos agrícolas (casca de arroz, bagaço de cana, parede de milho)}

Frella S. Garcia-Larreta I

frellas.sgarcia@gmail.com

Jhonny M. Vergara-Sanisaca II

jhonny.vergara@gmail.com

Mauro S. Nieto-Erazo III

dryago777@gmail.com

Mauro S. Nieto-Aguirre IV

mauro.nietoa@ug.edu.ec

Dolores B. Erazo-López V

dolores1@gmail.com

Correspondencia: dolores.erazol@ug.edu.ec

I. Magister en Diseño Curricular; Diplomado en Docencia Superior; Química y Farmacéutica;

Universidad de Guayaquil, Guayaquil, Ecuador.

II.

Químico y Farmacéutico, Universidad de Guayaquil, Guayaquil, Ecuador.

III.

Medico; Universidad de Guayaquil, Guayaquil, Ecuador.

IV . Medico; Centro de Salud Cisne 2, Guayaquil, Ecuador.

v. Magister en Bioquímica Clínica; Química y Farmacéutica; Universidad de Guayaquil, Guayaquil, Ecuador. 
Estudio comparativo del rendimiento del furfural a partir de diferentes residuos agrícolas (Cascarilla de Arroz, Bagazo de Caña, Zuro de Maíz)

\section{Resumen}

El futuro de los carburantes fósiles se está viendo afectado, pues las reservas de petróleos tienen a minimizarse a un futuro cercano. En el presente trabajo de titulación orienta a la implementación de métodos alternativos para la obtención de subproductos químicos a partir de los residuos agrícolas considerados como fuentes principales de reservas energéticas. De este modo se realiza un estudio comparativo del rendimiento de furfural a partir de la cascarilla de arroz, zuro de maíz, bagazo de caña, para la obtención de la molécula de furfural considerada como el mayor producto derivado de material lignocelulósico en los países desarrollados y utilizada como base para la síntesis de otros productos químicos. Cada residuos utilizado como materia prima es rica en pentosas componentes fundamentales para la síntesis de furfural obtenido a través de una destilación simple para posteriormente ser sometidos a ensayos cualitativos, estas pruebas se fundamentan en el que furfural forma derivados al reaccionar con ciertos reactivos tal es el caso de la 2-4 dinitrofenilhidrazina y el anilina en medio acético glacial ambas reacciones forman coloración en presencia de furfural. El procedimiento para el análisis cuantitativo de furfural incluye la utilización del equipo de cromatografía liquida de alta eficiencia acoplada a un detector UV (HPLC-UV). Los resultados obtenidos de la cuantificación determinan que el mayor contenido de furfural se obtiene de los desechos del zuro de maíz. Este tratado se enfoca en la producción de furfural a partir de las pentosas contenidas en los desechos del maíz, desecho agrícola pocos utilizados después de la cosecha. Los estudios para la implementación de biomasa para obtener productos químicos, ayudara a poner en marcha nuevas biorrefinerías en el país, obtener furfural ayudará a las industrias a sintetizar productos para que sean utilizadas en otras industrias químicas. Es importante que instituciones públicas y privadas promuevan el desarrollo y financiamiento proyectos cuyo objetivo es trabajar con residuos agrícolas para obtener fuentes alternas de energía.

Palabras claves: Rendimiento del furfural; residuos agrícolas; países desarrollados. 
Frella S. Garcia-Larreta; Jhonny M. Vergara-Sanisaca; Mauro S. Nieto-Erazo; Mauro S. Nieto-Aguirre; Dolores B.

\section{Abstract}

The future of fossil fuels is being affected because petroleum reserves have minimized the near future. In the present work aims to titration implementing alternative methods for obtaining chemical byproducts from agricultural waste considered as main sources of energy reserves. Thus a comparative study of the performance of furfural is made from rice husk, corn cob, bagasse, to obtain furfural molecule considered the largest product derived from lignocellulosic material in developed countries and used as the basis for the synthesis of other chemicals. Each waste used as raw material is rich in pentoses fundamental for the synthesis of furfural obtained through a simple distillation to be subsequently subjected to qualitative tests components, these tests are based on that form furfural derivatives by reaction with certain reagents as in the If the dinitrophenylhydrazine 2-4 and the aniline in glacial acetic medium both reactions form color in the presence of furfural. The procedure for quantitative analysis involves the use of furfural team high performance liquid chromatography coupled to a UV (HPLC-UV) detector. The results of the quantification determine that the highest content of furfural is obtained from waste corn cob. This treaty focuses on the production of furfural from pentose contained in corn waste, agricultural waste little used after harvest. Studies for implementation of biomass for chemicals, will provide implement new biorefineries in the country, furfural help industries to synthesize products so that they are used in other chemical industries. It is important that public and private institutions to promote the development and financing projects whose goal is to work with agricultural waste for alternative energy sources.

Key words: Furfural performance; agricultural waste; developed countries. 
Estudio comparativo del rendimiento del furfural a partir de diferentes residuos agrícolas (Cascarilla de Arroz, Bagazo de Caña, Zuro de Maíz)

\section{Introducción.}

Desde periodos antiguos y hasta la actualidad la biomasa es considerada un gran depósito de energía, fue uno de los primeros combustibles potentemente utilizado hasta que se inició la revolución industrial. El camino hacia el progreso en aquellos tiempos provocó que el aprovechamiento de la biomasa se viese desplazada por el uso masivo de los combustibles fósiles, en la actualidad la biomasa continúa siendo el primordial recurso energético poco aprovechado en el mundo (1).

El futuro de los combustibles fósiles se está viendo afectado a nivel mundialmente, se estima que las reservas petrolíferas tienen sus días contados, gracias al aumento de su explotación. Debido a su inestabilidad y progresivo agotamiento sumado a la gran demanda en el mundo y la emisión de gases contaminantes, ha provocado la búsqueda de nuevas alternativas para el desarrollo de combustibles, por esta razón los países desarrollados han considerado a los residuos agrícolas como materia prima idónea para la generación de productos químicos y energía, gracias a su carácter renovable y amplia distribución (2).

El presente trabajo tiene como objetivo realizar un estudio comparativo del rendimiento de furfural a partir de diferentes residuos agrícolas (zuro de maíz, bagazo de caña, cascarilla de arroz), considerados como principales productos de desechos generados de la actividad agricultora del país. Realizar un estudio de furfural para el Ecuador servirá para sintetizar derivados químicos y sustitutos del petróleo dentro del campo de la fibra, resinas y solventes aplicadas en la biorrefinerías que se encuentran en desarrollo en el país. Gracias al aumento progresivo y como consecuencia del agotamiento petrolífero, es importante la búsqueda de fuentes alternas al petróleo para obtener otros agregados químicos (3). 
Frella S. Garcia-Larreta; Jhonny M. Vergara-Sanisaca; Mauro S. Nieto-Erazo; Mauro S. Nieto-Aguirre; Dolores B.

Los residuos biomásicos utilizados como materias primas tienen un elevado contenido de pentosas para la producción de moléculas químicas como el furfural, mediante la hidrólisis ácida de las pentosas y una cuantificación se identificara cuál de estos residuos estudiados brindará un mayor rendimiento de furfural. La producción de esta molécula generará un desarrollo para la síntesis de otros productos químicos como es el caso de las resinas de molde, adhesivos para madera, fungicidas, aromatizantes, agentes vulcanizantés a nivel industrial en el país (4).

En el diseño de esta investigación se plantea la obtención de furfural a partir de zuro de maíz, bagazo de caña, cascarilla de arroz. Las pentosas contenida en estos residuos a través de una hidrolisis ácida se deshidrataran para la formación de furfural, mediante una destilación simple y Re destilación se obtendrá esta molécula que posteriormente se someterán a ensayos cualitativos con el reactivo de identificación de compuestos carbonilos, tal es el caso de la 2-4 dinitrofenilhidrazina y anilina en medio ácido, confirmando la presencia de este compuesto en cada destilados través de las pruebas cualitativas, se realizara una cuantificación en HPLC-UV quien determinara cuál de estos residuos agrícolas brindará un mayor contenido de furfural.

Los desechos de maíz utilizados representan la materia prima ideal para la producción de furfural por ser renovable y altamente producida en el país. El furfural es uno de los primordiales productos posibles a obtener en una refinería procesadora de biomasa, poner en funcionamiento estas industrias en el país, lo colocara al mismo nivel del resto de países que han iniciado estrategias de aprovechamiento industrial basado en la gestión de residuos agrícolas. (1)

El objetivo de la presente investigación es - Realizar un estudio comparativo del rendimiento de furfural a partir de diferentes residuos agrícolas (cascarilla de arroz, cachaza de caña, zuro de maíz). 
Estudio comparativo del rendimiento del furfural a partir de diferentes residuos agrícolas (Cascarilla de Arroz, Bagazo de Caña, Zuro de Maíz)

\section{Materiales y métodos.}

\section{Métodos teóricos inductivos}

Este método permitirá la recolección de información bibliográfica para la preparación del marco teórico, de los residuos agrícolas que se utilizaran y también de la molécula furfural que se obtendrá.

Mediante este proceso ayudara a interpretar datos empíricos y como final llegar a una conclusión en general.

Método empírico de experimentación científica:

En el presente trabajo se utilizó este método para ayudar a comprobar la hipótesis planteada dar respuesta a las preguntas científicas de la investigación tomando como base un conjunto de hechos y datos que se obtienen en los procesos. 
Frella S. Garcia-Larreta; Jhonny M. Vergara-Sanisaca; Mauro S. Nieto-Erazo; Mauro S. Nieto-Aguirre; Dolores B. Erazo-López

Diagrama de procesos de la metodología

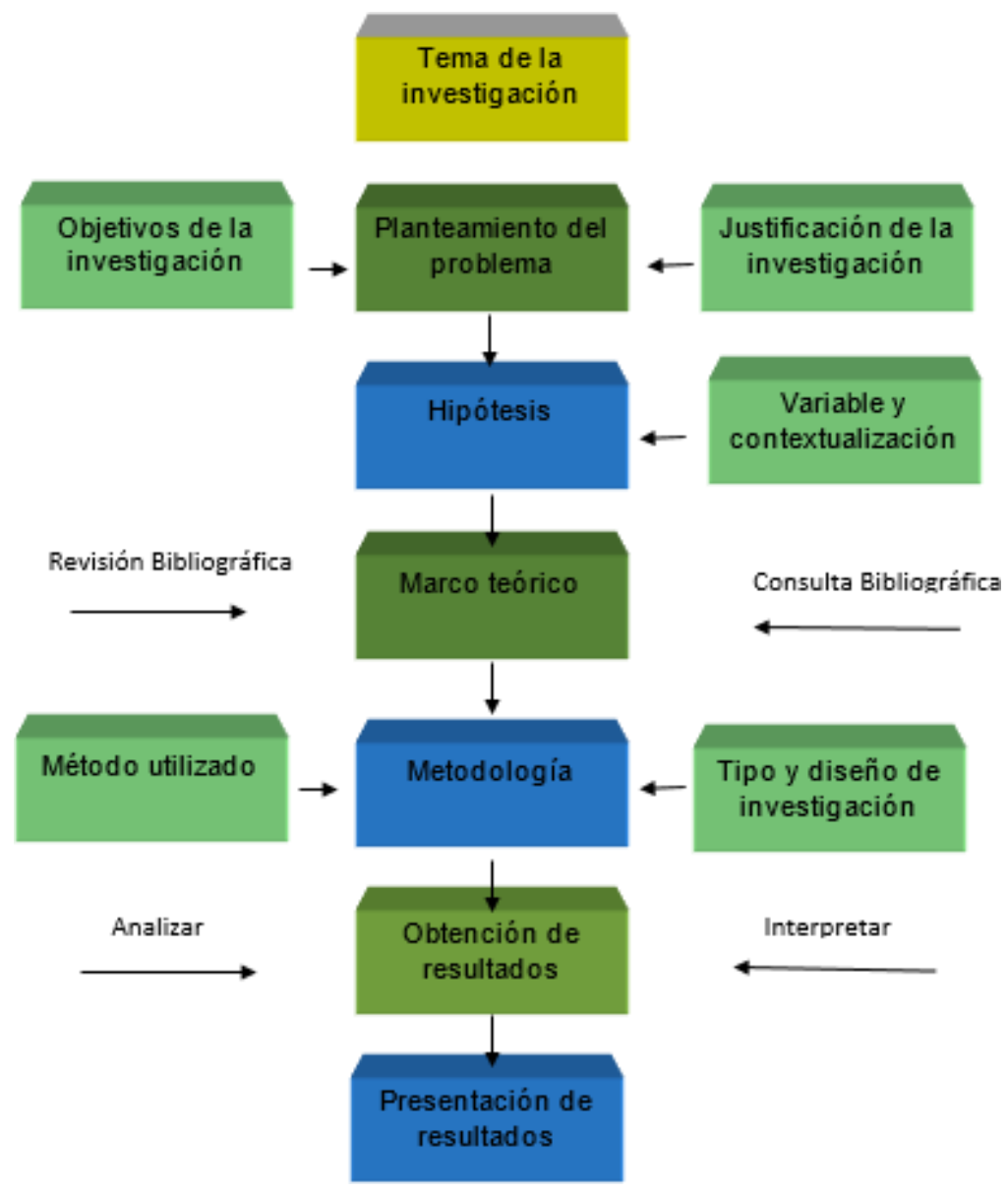


Estudio comparativo del rendimiento del furfural a partir de diferentes residuos agrícolas (Cascarilla de Arroz, Bagazo de Caña, Zuro de Maíz)

Diagrama de procesos de obtención de furfural

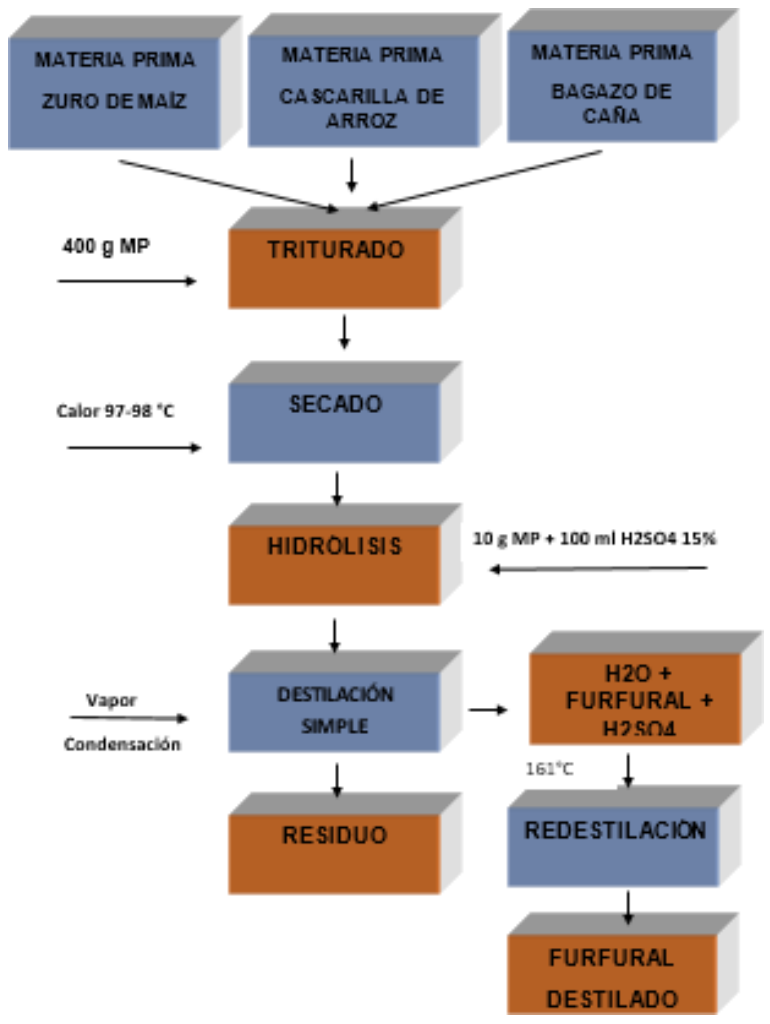

Diagrama de procesos de identificación cualitativa y cuantificación de furfural

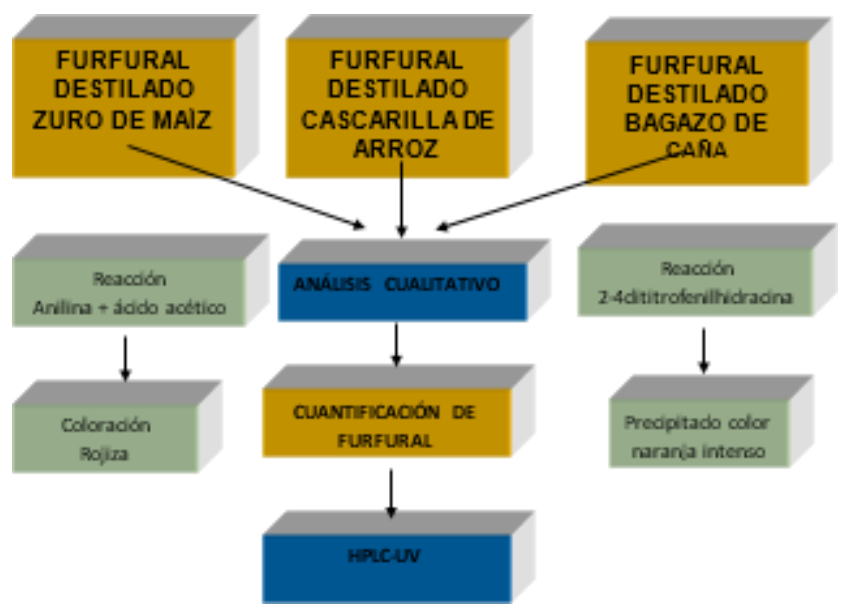


Frella S. Garcia-Larreta; Jhonny M. Vergara-Sanisaca; Mauro S. Nieto-Erazo; Mauro S. Nieto-Aguirre; Dolores B.

Tipo de investigación

En el presente trabajo el tipo de investigación que usaremos es de carácter exploratorio.

\section{Diseño de la investigación}

El diseño que se acopla al presente trabajo es experimental con un solo grupo, no se tiene grupo control, la medición de la variable se la realiza posteriormente. No se toma en consideración para este análisis variables externas.

\section{Recolección de datos}

Para la obtención de datos dentro del presente trabajo de titulación se basó en:

La observación de los ensayos las cuales fueron aplicadas en las pruebas preliminares, como prueba orientativa de identificación de furfural, obteniendo de esta manera los datos cualitativos.

Pruebas e inventarios estandarizados, a través de este método de recolección de datos se realizó la determinación de contenido de humedad de los residuos bajo los métodos de la AOAC:

AOAC 19TH 945,38 B método utilizado para determinar humedad en cereales y derivados

AOAC 19TH 925.45 método utilizado para determinar humedad en azucares y derivados.

La cuantificación de furfural se realizó bajo el método de Robert R.M. y sus colaboradores 1978 mediante HPLC-UV, bajo las siguientes condiciones cromatografícas. 
Estudio comparativo del rendimiento del furfural a partir de diferentes residuos agrícolas (Cascarilla de Arroz, Bagazo de Caña, Zuro de Maíz)

\section{Resultados.}

\begin{tabular}{|}
$\mid$\begin{tabular}{|c|c|c|c|}
\hline \multirow{2}{*}{ ZURO DE MAÍZ Z } & PARAMETRO & RESULTADO & UNIDAD \\
\cline { 2 - 4 } & HUMEDAD & 75,21 & $\mathrm{~g} \%$ \\
\hline \multirow{3}{*}{ BAGAZO DE CAÑA } & PARAMETRO & RESULTADO & UNIDAD \\
\cline { 2 - 4 } & HUMEDAD & 67,28 & $\mathrm{~g} \%$ \\
\hline \multicolumn{3}{|c|}{} \\
\hline \multirow{2}{*}{ CASCARILLA DE Arroz } & PARAMETRO & RESULTADO & UNIDAD \\
\cline { 2 - 4 } & HUMEDA & 13,63 & $\mathrm{~g} \%$ \\
\hline
\end{tabular}
\end{tabular}

\section{Caudro $N^{\circ}$ 1.- Contenido de Humedad de los Residuos Agrícolas}

El contenido de humedad de los residuos en estudio fue realizado bajo el método de la AOAC donde se demuestra que existe una mayor concentración de humedad entre los residuos del zuro de maíz y bagazo de caña, cotejados con el bajo porcentaje de humedad hallada en la cascarilla de arroz, este último residuo se observa físicamente su bajo contenido de humedad motivo por el cual es expuesto al sol tras su cosecha. Hay que tomar en consideración este parámetro de humedad con el objetivo de eliminar interferencia de agua durante la digestión ácida de la muestra.

\begin{tabular}{|c|c|}
\hline MATERIAL & PENTOSAS \\
\hline Mazorca / Zuro de Maíz & 30 a $32 \%$ \\
\hline Cascarilla de arroz & $25 \%$ \\
\hline Bagazo de caña & $18 \%$ \\
\hline $\begin{array}{c}\text { Nota: El contenido de pentosas se calcula mediante la conversión del residuo a furfural } \\
\text { y posterior cuantificación del producto obtenido, generalmente por precipitación con } \\
\text { ácido barbitúrico Fuente (5). }\end{array}$ \\
\hline \multicolumn{2}{|c}{} \\
\hline
\end{tabular}

Cuadro $N^{\circ}$ 2.- Contenido teórico de Pentosas de los Residuos Agrícolas 
Frella S. Garcia-Larreta; Jhonny M. Vergara-Sanisaca; Mauro S. Nieto-Erazo; Mauro S. Nieto-Aguirre; Dolores B.

\section{Contenido teórico de Pentosas de los Residuos Agrícolas}

El contenido teórico de pentosas de los residuos utilizados en el presente trabajo, cuyo mayor porcentaje se concentra en el zuro de maíz con un 32\% de pentosas. Estos tres residuos agrícolas son las fuentes principales de desecho que se generan tras la cosecha y poco utilizados a nivel industrial. El contenido de pentosas se mide mediante la conversión de éstas a furfural y una posterior cuantificación del producto obtenido, generalmente por precipitación con ácido barbitúrico. Cuando las condiciones experimentales empleadas son correctas, el rendimiento del furfural por este procedimiento ha comprobado que es del 100\% (6).

\section{Resultados obtenidos de ensayos preliminares.}

\section{Análisis Cualitativo de Precipitación con el Reactivo de la 2-4 Dinitrofenilhidrazina}

\begin{tabular}{|c|c|c|c|c|c|c|}
\hline \multirow{2}{*}{\multicolumn{3}{|c|}{ Análisis Cualitativo }} & \multicolumn{4}{|c|}{ Reacción 2-4 Dinitrofenilhidrazina } \\
\hline & & & \multicolumn{4}{|c|}{ PRECIPITACIÓN } \\
\hline Residuos & $\mathrm{N}^{\circ}$ Destilados & VOL. Destiladd & \# ENSAYOS & Alta & Media & Baja \\
\hline \multirow{4}{*}{ Zuro de maíz } & \multirow{2}{*}{ Destilado A } & \multirow{2}{*}{$110 \mathrm{ml}$} & $\mathrm{A} 1$ & $x$ & & \\
\hline & & & $\mathrm{A} 2$ & $x$ & & \\
\hline & \multirow{2}{*}{ Destilado B } & \multirow{2}{*}{$105 \mathrm{ml}$} & B1 & $x$ & & \\
\hline & & & B2 & $x$ & & \\
\hline \multirow{4}{*}{ Cascarilla de Arroz } & \multirow{2}{*}{ Destilado A } & \multirow{2}{*}{$100 \mathrm{ml}$} & A1 & & $\mathrm{x}$ & \\
\hline & & & $\mathrm{A} 2$ & & $\mathrm{x}$ & \\
\hline & \multirow{2}{*}{ Destilado B } & \multirow{2}{*}{$100 \mathrm{ml}$} & B1 & & $\mathrm{x}$ & \\
\hline & & & B2 & & $\mathrm{x}$ & \\
\hline \multirow{4}{*}{ Bagazo de Caña } & \multirow{2}{*}{ Destilado A } & \multirow{2}{*}{$90 \mathrm{ml}$} & $\mathrm{A} 1$ & & & $x$ \\
\hline & & & $\mathrm{A} 2$ & & & $x$ \\
\hline & \multirow{2}{*}{ Destilado B } & \multirow{2}{*}{$95 \mathrm{ml}$} & B1 & & & $x$ \\
\hline & & & B2 & & & $\mathrm{x}$ \\
\hline
\end{tabular}

De cada residuo agrícola en estudio se realizó una destilación por duplicado obteniendo dos destilados A Y B. El reactivo de la 2-4dinitrofenilhidracina forma un precipitado color naranja en presencia de compuestos carbonilos. La muestra del zuro de maíz demostró un alto contenido de 
precipitado en comparación con la cascarilla de arroz y la poca visualización de un precipitado en el bagazo de caña. De cada destilado A y B se realizaron las pruebas por duplicado.

\section{Análisis Cualitativo de Coloración con el Reactivo de Anilina en Medio Ácido}

\begin{tabular}{|c|c|c|c|c|c|c|}
\hline \multirow{2}{*}{\multicolumn{3}{|c|}{ Análisis Cualitativo }} & \multicolumn{4}{|c|}{ Anilina + Ácido Acetico } \\
\hline & & & \multicolumn{4}{|c|}{ COLORACIÓN } \\
\hline Residuos & $\mathrm{N}^{\circ}$ Destilados & VOL. Destiladc & \# ENSAYOS & Alta & Media & Baja \\
\hline \multirow{4}{*}{ Zuro de maíz } & \multirow{2}{*}{ Destilado A } & \multirow{2}{*}{$110 \mathrm{ml}$} & $\mathrm{A} 1$ & $x$ & & \\
\hline & & & $\mathrm{A} 2$ & $x$ & & \\
\hline & \multirow{2}{*}{ Destilado B } & \multirow{2}{*}{$105 \mathrm{ml}$} & B1 & $x$ & & \\
\hline & & & B2 & $x$ & & \\
\hline \multirow{4}{*}{ Cascarilla de Arroz } & \multirow{2}{*}{ Destilado A } & \multirow{2}{*}{$100 \mathrm{ml}$} & A1 & & $x$ & \\
\hline & & & $\mathrm{A} 2$ & & $x$ & \\
\hline & \multirow{2}{*}{ Destilado B } & \multirow{2}{*}{$100 \mathrm{ml}$} & B1 & & $x$ & \\
\hline & & & B2 & & $x$ & \\
\hline \multirow{4}{*}{ Bagazo de Caña } & \multirow{2}{*}{ Destilado A } & \multirow{2}{*}{$90 \mathrm{ml}$} & A1 & & & $x$ \\
\hline & & & $\mathrm{A} 2$ & & & $x$ \\
\hline & \multirow{2}{*}{ Destilado B } & \multirow{2}{*}{$95 \mathrm{ml}$} & B1 & & & $x$ \\
\hline & & & B2 & & & $x$ \\
\hline
\end{tabular}

De los destilados obtenidos con anterioridad se desarrolló la segunda prueba de identificación de compuestos carbonilos, esta reacción se basa en la formación de una coloración rojo cereza durante esta prueba. La intensidad de la coloración para el destilado de zuro de maíz fue alta comparada con la intensidad media presente en el destilado de la cascarilla de arroz y la baja tonalidad en los destilados de bagazo de caña. De cada destilado A y B se realizaron las pruebas por duplicado. 
Frella S. Garcia-Larreta; Jhonny M. Vergara-Sanisaca; Mauro S. Nieto-Erazo; Mauro S. Nieto-Aguirre; Dolores B.

\section{Resultados de la cuantificación de furfural.}

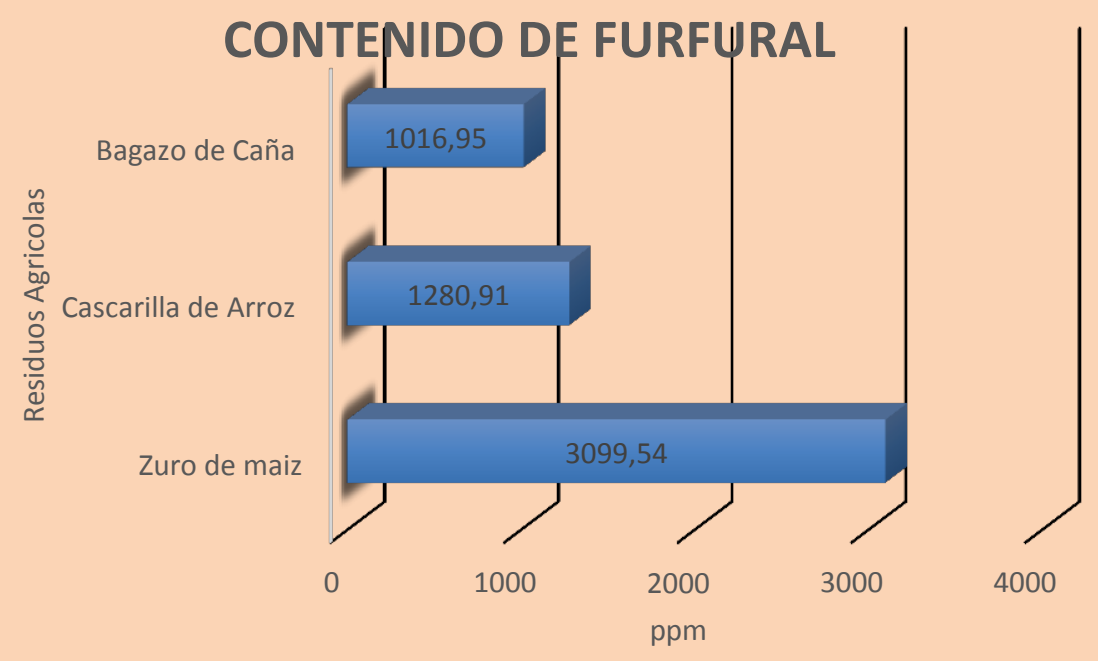

Grafico $N^{\circ}$ 1.- Resultados del Contenido de Furfural en los Residuos Agrícolas determinados en

\section{HPLC-UV}

El mayor contenido de furfural de los residuos agrícolas, cuyas determinaciones fueron bajo HPLC-UV. El mayor contenido de furfural se encuentra en los residuos agrícolas provenientes del zuro de maíz con concentraciones de $3099.54 \mathrm{mg} / \mathrm{kg}$ de furfural cuyo resultado se coteja con el contenido de pentosa que se demuestra en el grafico I con u porcentaje de pentosas para el zuro de maíz de $32 \%$, la segunda mayor concentración se encuentran en los residuos provenientes de la cascarilla de arroz con concentraciones de1280.91 mg/kg de furfural y el menor contenido de furfural se encuentra en los residuos de bagazo de caña cuyos resultados se cotejan con el bajo contenido de pentosas para este residuos. 


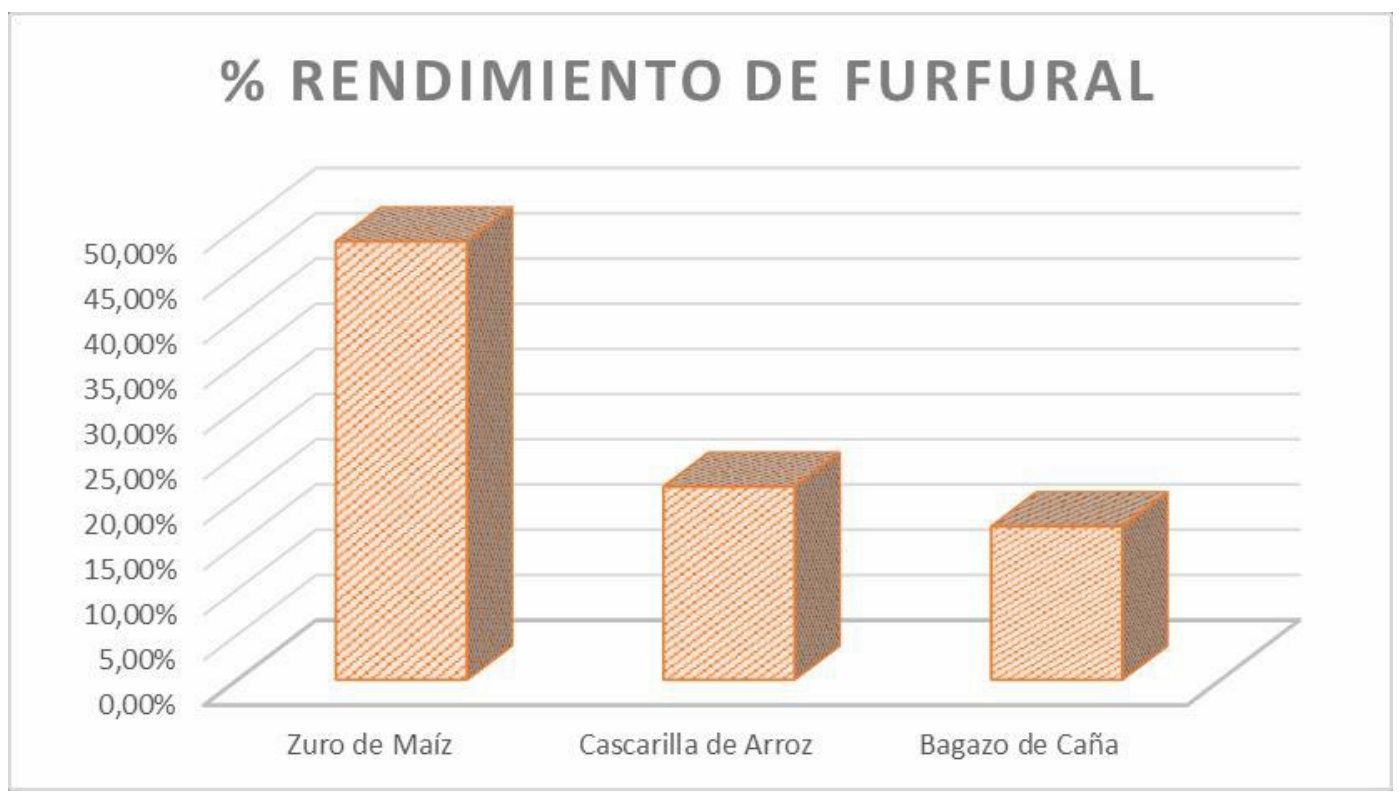

\section{Grafico $N^{\circ}$ 2.- Rendimiento de Furfural de cada materia prima}

De acuerdo con los cálculos de estequiometria, se demuestra que el residuo de mayor rendimiento de furfural tras su destilación a nivel de laboratorio, se encuentran en el zuro maíz con un $50 \%$ de rendimiento comparados con los bajos rendimientos de la cascarilla de arroz $21.94 \%$ y $16.94 \%$ del bagazo de caña. A pesar de poseer la mitad del rendimiento hay que tomar en consideración ciertos parámetros que pueden afectar la productividad, como condiciones de presión, temperatura e impurezas de los reactivos durante la destilación, almacenamiento de muestras para su análisis.

\section{Conclusiones.}

Los estudios teóricos del contenido de pentosas determinaron que el zuro de maíz presenta un rendimiento de pentosas del 30 a $32 \%$, la cascarilla de arroz con un contenido de pentosas del $25 \%$ y el bagazo de caña $18 \%$ de pentosas, dichos datos permite tener una orientación sobre el rendimiento de furfural durante la destilación. 
Frella S. Garcia-Larreta; Jhonny M. Vergara-Sanisaca; Mauro S. Nieto-Erazo; Mauro S. Nieto-Aguirre; Dolores B. Erazo-López

La destilación simple de cada muestra (zuro de maíz cascarilla de arroz, bagazo de caña) se obtuvo destilados color transparentes con olor característico a almendra.

La reacción con 2-4 dinitrofenilhidrazina demostró la presencia de furfural formando un precipitado color naranja característico de esta reacción en cada uno de los destilados. En la muestra de zuro de maíz se presentó una coloración y un precipitado más intensa, en comparación con la muestra de cascarilla de arroz y bagazo de caña. De la misma manera dicha intensidad de color presente el zuro de maíz se halló al hacer reaccionar con anilina en medio acético formando una coloración roja intensa en los destilados, Por medio de estas reacciones se ratifica la presencia de furfural en cada uno de los destilados.

La cuantificación de furfural mediante (HPLC-UV) demostró la presencia de furfural en los tres residuos agrícolas, sin embargo el residuo biomásico que mayoritariamente posee furfural proceden de los desechos del zuro de maíz con un contenido de $3099.54 \mathrm{mg} / \mathrm{kg}$.

\section{Bibliografia.}

1. Amaya Juárez FR, Flores Solano OD. Estudio de Ingenieria Sostenible para la obtención de furfuralL como subproducto de la Agroindustria del Maíz. Ingeniero Químico. San Salvador: Universidad de El Salvador, Escuela de Ingeniería Química; 2011.

2. Martín Gil J. El futuro de los biocombustibles: biorrefinerías integradas. Discursos universitariosEspaña-Valladolid. Valladolid: Universidad de Valladolid; 2009 Septiembre 30.

3. Plaza Canchingre EL, Posligua Chica EP. Obtención de Furfural a partir de la Cascarilla de Arroz. Guayaquil: Universidad de Guayaquil, Facultad de Ingeniería Química; 2006 Julio.

4. Sádaba Zubiri I, Manuel López G. Catalizadores para biorrefinería: obtención de furfural y su transformación a productos de condensación aldólica. Tesis de Doctorado. Madrid: Universidad autonoma de Madrid; 2012 Jan 20.

5. O”Brien P. Furfural Chemical and Biofuels from Agriculture. [Online]. Australia; 2006 [cited 2015 Julio 23 
Estudio comparativo del rendimiento del furfural a partir de diferentes residuos agrícolas (Cascarilla de Arroz, Bagazo de Caña, Zuro de Maíz)

6. Zeitsch K. The chemistry and technology of furfural and its many by-products. 13th ed. Germany: Elsevier Science; 2000. 